(From the Hull Physiological Laboratory of the University of Chicago.)

\title{
Ueber \\ die physiologische Wirkung von Alkalien und Säuren in starker Verdünnung.
}

Von

Jacques Loeb.

Bekanntlich kann die Bewegung von Spermatozoen oder von Cilien, sobald sie dem Erlöschen nahe ist, dadurch wieder hervorgerufen werden, dass wir Alkalien in starker Verdünnung zufügen. Man sagt, dass das Alkali erregend wirke. Das aber erklärt die Sache nicht, da wir das Wort Erregung immer dann in der Physiologie anwenden, wenn eine Thätigskeitszunahme stattfindet, gleichviel, was die Ursache und Mechanik dieser Thätigkeitszunahme ist.

Wenn wir zu einer Mechanik dieser Wirkung der Alkalien vordringen wollen, so müssen wir die Frage aufwerfen, wie dieselben in den Chemismus oder die Energetik der Lebenserscheinungen eingreifen. Hierbei werden wir aber bald auf die bekannte Thatsache geführt, dass Alkalien einen fördernden Einfluss auf die Sauerstoffübertragung und damit die Oxydationsvorgänge in thierischen Geweben haben. Entsprechend haben Säuren den entgegengesetzten Effect. Es wäre daher denkbar, dass die alte Beobachtung über den angeblich "erregenden" Einfluss der Alkalien auf die Flimmerbewegungen darauf zurückzuführen sei, dass der Zusatz von Alkali eine Zunahme der Oxydationsvorgänge und damit der Energieentwicklung bestimme.

Um diese?Vermuthung zu prüfen, schlug ich Herrn Dr. Zoethout vor, zu versuchen, ob bei langsamer Sauerstoffentziehung die Flimmerbewegung nicht früher in einem neutralen als in einem alkalischen Medium zum Stillstand käme. Zum Studium der Flimmerbewegung wurden bewimperte Infusorien (Paramaecien) benutat. Dieselben wurden in destillirtes Wasser gebracht und entweder in diesem beobachtet, oder es wurde dem destillirten Wasser etwas Säure oder 
Alkali zugesetzt. Dann wurden alle drei Lösungen (oder zwei derselben) in Engelmann'sche Kammern gebracht und gleichzeitig demselben nicht zu starken Wasserstoffstrom ausgesetzt. Der Wasserstoff wurde sorgfältig in zwei Waschflaschen mit Alkali, eine mit übermangansaurem Kali und eine mit Wasser ausgewaschen, ehe er in die Engelmann'sche Kammer eintrat. Es ergab sich, dass Alkalien ( $\mathrm{NaHO}$ ) in schwacher Verdünnung die Lebensdauer der Paramaecien bei Sauerstoffentziehung erheblich vermehren. Allein die Verdünnung der Alkalilösung muss in diesen Fällen sehr gross sein. Eine Alkalilösung ( $\mathrm{NaHO}$ ) von ${ }^{1 / 1200} \%$ bis ${ }^{1 / 1600} \%$ verlängerte die Lebensdauer der Paramaecien bei langsamer Sauerstoffverdrängung um 100 bis $200 \%$ ! Bei stärkerer Verdïnnung war das Alkali wirkungslos, bei geringerer Verdünnung beschleunigte es den Tod der Paramaecien. Säuren waren in jeder Verdünnung schädlich, schon eine $1 / 1600 \%$ ige Lösung von $\mathrm{HCl}$ beschleunigte das Ende. Es wurden viele Versuche angestellt. Sie fielen so eindeutig und schlagend aus, wie man es nur wünschen kounte.

Wir wissen, dass im Fieber die Alcalescenz des Blutes herabgesetzt ist. Ich habe daran gedacht, ob dieser Umstand nicht dazu beitragen könnte, den Substanz- und Kräftezerfall, der für das Fieber charakteristisch ist, herbeizuführen. Die verringerte Alcalescenz des Blutes muss zu einer Verringerung der Oxydationsvorgänge in den Geweben führen. Die Spaltungsvorgänge würden damit mehr in den Vordergrund treten. $\mathrm{Ob}$ diese Argumentation richtig ist oder nicht, ich schloss, dass die Paramaecien bei hoher Temperatur länger leben, wenn sie in einer schwach alkalischen Lösung sind, als wenn sie in neutraler oder gar saurer Lösung sich befinden, und die Versuche. die Herr Dr. Zoethout hierüber ausführte, bestätigten meinen Schluss. Bei Zusatz von Alkali (in derselben Concentration, wie bei Sauerstoffentziehung) lebten und bewegten sich die Paramaecien länger in einer Temperatur von $40^{\circ}$ als in neutraler oder gar saurer Lösung.

Um schliesslich die letzten Bedenken zu beseitigen, schlug ich Herrn Dr. Zoethout vor, Versuche darüber anzustelles, ob die giftige Wirkung von Cyankalium durch Alkali verringert werden könnte. Wir wissen ja durch die Arbeiten von Claude Bernard und Geppert, dass Cyankalium dadurch tödtet, dass es die Sauerstoffaufnahme in den Geweben unmöglich macht. Wenn es nun gelänge, durch Zusatz von einer Spur von Alkali die giftigen Wir- 
kungen von Cyankalium zu verringern, so würde damit eine neue Stütze geliefert für den Gedanken, dass die angeblich erregende Wirkung des Alkalis nur indirect erfolgt, nämlich dadurch, dass es die Sauerstoffübertragung in den Geweben und damit die Energieentwicklung steigert. Der Versuch wurde in folgender Weise angestellt: Ein Tropfen einer 1\% igen KNC-Lösung wurde zı zehn Tropfen Wasser mit Paramaecien zugefügt. In einer solchen Lösung gingen die Organismen bald zu Grunde. Fügte man aber Alkali hinzu, so dass die Alkalilösung weniger als $1 / 200 \%$ stark war, so blieben die Paramaecien 1/2- bis 3 mal so lange am Leben. War die Alkalilösung stärker, so beschleunigte sie den Tod. Säuren wirkten wieder in jedem Verdünnungsgrad schädlich.

Es fragte sich nun, ob andere Gifte sich ebenso verhielten. Das war der Fall mit Atropin. Schwaches Alkali vermehrte die Widerstandsfähigkeit der Paramaecien gegen Atropin, Säuren verminderten die Widerstandsfähigkeit. Ganz anders dagegen war der Einfluss der Alkalien bei Anwendung anderer Gifte. Bei Vergiftung mit Strychnin erwies sich Alkali wie Säure in jeder Verdünnung als schädlich, vorausgesetzt dass es überhaupt noch eine Wirkung übte. Auch bei Vergiftung mit Veratrin konnte die Lebensdauer der Paramaecien nicht verlängert werden durch Zufügung schwacher Dosen von Alkali. Diese Versuche werden fortgesetzt.

Ich selbst stelite nun eine andere Versuchsreihe an, um die Wirkung der Alkalien und Säuren zu analysiren. Ich ging von der Annahme aus, dass bei Vorgängen des embryonalen Wachsthums und vielleicht auch bei der Entwicklung synthetische Vorgänge. in ausgedehntem Umfange stattfinden müssen. Nach Hoppe-Seyler ist es aber wesentlich die von Sauerstoff stammende Energie, welche Synthesen im Thierkörper ermöglicht. Es ist ja übrigens anch direct erwiesen, dass, von wenigen Anaeroben abgesehen, ohne freien Sauerstoff Wachsthum und Entwicklung sehr bald zum Stillstand kommen. Ich versuchte desshalb, ob es nicht möglich sei, durch Zusatz von schwachem Alkali Entwicklung und Wachsthum von Embryonen zu beschleunigen. Die Versuche stellte ich an Seeigellarven an. Es ergab sich, dass Zusatz von $1 \frac{1 / 2-2}{2}$ cem einer $1 / 10$ normalen NaHOLösung zu $100 \mathrm{ccm}$ Seewasser die Fntwicklung und das Wachsthum der Seeigellarven so erheblich beschleunigt, dass man kaum glauben sollte, dass man es im normalen Seewasser und in alkalischem Seewasser mit Individuen derselben Cultur zu thun hat. (Bei Zusatz 
Ueb. d. phyșiol. Wirkung von Alkalien u. Sätren in starker Verdünnung. 425

von 2 ccm NaHO zu Seewasser entsteht ein Niederschlag von Magnesiumhydroxyd und Calciumhydroxyd. Filtrirt man, so findet man, dass noch ca. 1,4 cem der 1/10 norm. NaHO-Lösung in Lösung bleibt.) Bei Zusatz von weniger Alkali beobachtet man keine Wirkung. Zusatz von mehr Alkali vermehrt im Wesentlichen nur den Niederschlag im Seewasser. Säuren verzögern in jeder Concentration Entwicklung und Wachsthum. Bereits Zusatz von $1 \mathrm{ccm}$ einer $1 / 10$ norm. $\mathrm{HCl}$-Lösung zu $100 \mathrm{ccm}$ Seewasser verzögert die Entwicklung ganz erheblich. Aehnliche Versuche erhielt ich an Fischen, im Süsswasser wie im Seewasser. Ich glaube, dass wir daraufhin wohl schliessen dürfen, dass Alkalien in sehr verdünnter Lösung (etwa $1 / 1000-1 / 500$ - normale $\mathrm{NaHO}$-Lösung) die Oxydationsvorgänge in thierischen Geweben zu beschleunigen im Stande sind, während verdünnte Säuren die entgegengesetzte Wirkung haben.

Aus dem Gesagten ergeben sich eine Reihe von Problemen, die auch für die Praxis von Bedeutung werden dürften. Die erste Frage ist die, ob es sich nicht von Vortheil erweisen würde, wenn man in allen Fällen von Kochsalztransfusion, namentlich aber nach starkem Blutverlust, der Lösung etwas Alkali zufügen würde. Da für die oxydationsbeschleunigende Wirkung des Alkalis eine ausserordentlich schwache Concentration des Alkali nicht nur ausreichend, sondern geboten ist, so braucht man sich vor Nebenwirkungen des Alkalis nicht zu fürchten. Wie die Paramaecien bei O-Mangel länger am Leben bleiben, wenn ihnen Alkali geboten wird, so dürfte auch im Falle von grossen Blutverlusten der Zusatz von einer Spur Alkali bewirken, dass die Oxydationen trotz des herrschenden O-Mangels in den Geweben doch noch eine für die Erhaltung des Lebens ausreichende Intensität behalten. Man hat gelegentlich kohlensaures Natron der Kochsalzlösung bei Transfusionen zugefügt. Das ist durchaus rationell. Es ist nur noch der directe Nachweis zu bringen, dass gerade die Hydroxylionen in diesen Fällen das Werthvolle sind, dass sie eine oxydationserhöhende Wirkung haben, und es ist zu ermitteln, welches das Optimum der Verdünnung ist, in dem sie angewendet werden müssen.

Eine zweite Frage würde die sein, ob es sich nicht empfiehlt, im Fieber Alkali zu geben, um die Abnahme der Alcalescenz des Blutes zu verhindern resp. den schädlichen Wirkungen dieser Abnahme entgegen zu wirken. Wenn die Lebensdauer der Paramaecien bei hoher Temperatur durch Zusatz von Alkalien vermehrt 
426 Jacques Loeb: Ueber die physiologische Wirlsung von Alkalien etc.

wird, so dürfte es auch wohl möglich sein, dass in hohem, langandauerndem Fieber die Widerstandsfähigkeit und Lebensdauer menschlicher Gewebe verlängert wird. Mit Versuchen zur Lösung dieser Probleme sind wir gegenwärtig beschäftigt. Ueber das Ergebniss werden wir an dieser Stelle berichten. Die ausführliche Arbeit von Dr. Zoethout wird im American Journal of Physiology, und der Bericht über meine Versuche an Seeigellarven wird in der Zeitschrift für Entwicklungsmechanik erscheinen. Ueber andere Wirkungen der Hydroxylionen, die bei stärkerer Concentration auftreten, habe ich in meinen Arbeiten über Ionenwirkungen bereits angefangen zu berichten (Pflüger's Archiv Bd. 69 und 71). 